\title{
A design methodology for additive manufacturing applied to fused deposition modeling process
}

\author{
Boyard Nicolas $^{1}$, Christmann Olivier ${ }^{1, *}$, Rivette Mickael $^{2}$, and Richir Simon ${ }^{1}$ \\ ${ }^{1}$ LAMPA, Arts et Métiers ParisTech, 2 Boulevard du Ronceray, 49000 Angers, France \\ ${ }^{2}$ LCFC, Arts et Métiers ParisTech, 4 rue Augustin Fresnel, 57000 Metz, France
}

Received: 24 November 2015 / Accepted: 6 June 2019

\begin{abstract}
The aim of this article is to propose a design methodology for the production of parts in additive manufacturing (AM). The AM process allows new features (e.g., multi-material, fixed assemblies, complex shapes), and this paradigm shift requires the accompaniment of designers to take account of these characteristics. In response to this problematic, we propose a design methodology, in three stages, which respects the integrity of the digital channel and whose purpose is to provide a digital mock-up sliced ready to be manufactured on the most common AM process (Fused Deposition Modeling). From the specifications and the process knowledge, our methodology provides the designer a first solid geometry which satisfies all the constraints. Then, a topology optimization limits the useful volume of material of the part in order to limit the weight and the manufacturing time. If necessary, an optimized support providing manufacturability of the part is generated according to the same criteria. The methodology we propose is applied to a real industrial part.
\end{abstract}

Keywords: Design methodology / DFAM / support / CAD / FDM

\section{Introduction}

\subsection{Additive manufacturing}

Conventional manufacturing processes, such as machining, molding and plastic deformation, usually set up unique materials, known as homogeneous, linear and isotropic. Thus, from the specifications, the design of a part consists in defining its geometry, assigning a material to satisfy the mechanical constraints and finally to establish a manufacturing sequence. The limitations of current processes are pushed back by the additive manufacturing process (AM) which offers three new potentialities compared to conventional manufacturing processes, namely the realization of multimaterials part [1,2], achieving fixed assemblies [3] and the realization of hollow shapes with complex geometries [4].

The AM imposes several specific rules which the designer must absolutely take into account. These rules are complex because they are all interconnected. While the FDM process allows the use of many materials, the parts produced cannot generally claim the same strength as parts produced using conventional processes, due to the anisotropy induced by layer-by-layer printing [5], as

\footnotetext{
e-mail: Olivier.Christmann@ensam.eu
}

has been demonstrated, for example, in the case of injection molded parts. [6]. The FDM process is quite complex, because the different manufacturing parameters on which it is possible to play to ensure the strength of the part produced often have contradictory effects [7]. Basically, the mechanical strength depends on both the geometry of the part, the material used, but also the orientation of the part during the design. However, the orientation of the part depends on the desired surface finish and the working volume of the machine. And the working volume of the machine depends on the materials the machine could use. Of course, the orientation of the part is not the only variable that affects its resistance: the manufacturing parameters are also important. Many studies focus on studying the influence on tensile and compressive strength of different manufacturing parameters (e.g., [8-12]): air gap, layer thickness, raster angle, raster width, deposition speed, temperature, .... Mesoscale factors like the pattern and the infill percentage can also influence the strength of the part produced. Fernandez-Vicente et al. [13] showed that the pattern do not have a predominant influence, contrary to the inflill percenttage. They determined, within the framework of their study, that a filling of $100 \%$ allows a greatest tensile strength.

Conventional processes are widely formalized and documented and there is a large number of methodologies whose relevance varies with the design goals. Depending 
on the area and the application case, the formalism of design tools may change, however, the model proposed by Howard et al. [14] presents the general structure of the design process of the part. This model is based on the comparison and analysis of 23 design processes. It resumes the models of $[15,16]$ and the model VDI-2222 [17]. These design methodologies follow a downward pattern, from the analysis of the specifications to detailed design, to obtain a final geometry. Among the sequential methodologies and integrated methodologies, only the latter are sufficiently flexible to allow the interdependence of design choices. In particular, the Design For Manufacturing (DFM), which aims to optimize the design of a part according to the manufacturing process, seems to be suitable for the AM.

\subsection{A new design methodology for DFAM}

However, most of these methods are restricted to a vision based on constraints. The process is selected depending on the geometry of the part without taking advantage of this specific process, which is contrary to the principle of AM. Several research projects have been conducted to propose a design methodology dedicated to AM. The novelty is to design a part in accordance with all the advantages offered by this process.

A first iteration consisted in the proposal of redesign models dedicated to AM [18-20] for which the designer has a CAD mock-up to optimize. But the redesign of a part involves an upstream design work, and the result does not meet necessarily the specifications. Although this work is necessary in the case of non-compliant parts, it involves a high cost in design time. In addition, the redesign of a part is based in most cases on the previous geometry. Design freedoms are thus restricted by the previous choices of geometry, manufacturing or assembly.

If it is possible to start from the definition of specifications, we consider that it is a design work. Indeed, although the purpose is to redesign an existing part, the result is controlled neither by the geometry of the part, or by the manufacturing process, or by the material used. Currently, only Ponche [21] provides a solid basis for an AM oriented design methodology. However, this work is strongly oriented on powder projection material deposition process and it still faces some limitations: the material is imposed by the specifications, no step allows the validation of the part before the phase of topological optimization, it is not possible to change the result of topological optimization and the support is not taken into account. However, regarding manufacturing time, it may be advantageous to modify the geometry of the part in order to limit the presence of the support.

We therefore wish to propose a new design methodology compliant with Design for Additive Manufacturing, offering expanded structure, taking into account the specificities of the AM and a pertinent analysis of the part during the design. Our methodology is characterized by a larger number of design choices, such as the management of Young's modulus, the validation of the solid via a finite element analysis and the optimization of the support. As part of the work reported in this article, we will not consider all the manufacturing parameters because we

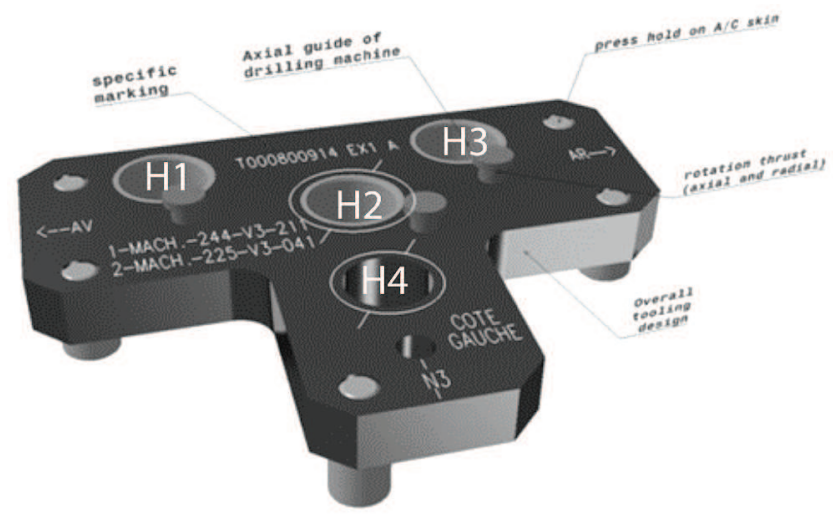

Fig. 1. CAD Mock-up of the existing drilling grid.

want this methodology to be integrated into a tool that is easy to use and saves the designer time. The parameters specific to the manufacturing process are quite complex to understand, and can have opposing effects, requiring compromises to be found. These considerations are also still the subject of research, and the reader may for example refer to the article [22] assessing the impact of FDM process parameters on the strength of the built parts by using Taguchi's design of experiments (DOE), using the same industrial case study. Nevertheless, the automation of our methodology may make it possible to integrate new manufacturing parameters. For the specific case, we mainly consider the orientation of the part (which can also influence the surface condition), the thickness of the internal and external surfaces and the internal filling. Even if we will mention the problem of warping due to cooling of the lower layers in the FDM process, we do not fully integrate it into our methodology because even if it is possible to modify the internal structure of the part [23], there are guidelines to limit this effect as much as possible and which are therefore more good practices than parameters to be taken into account. These good practices will be addressed in the discussion.

Ultimately, the aim of the methodology is to produce a mock-up ready to be sliced and manufactured on an AM machine with the functional expression of the need as a starting point, while taking into account the manufacturing process. Our methodology is centered on the generation, in successive stages, of a CAD mock-up.

\section{A Methodology for additive manufacturing}

\subsection{An industrial case study}

To illustrate and validate the methodology we propose, we chose to work on a real case study provided by a manufacturer, which corresponds to a drilling grid for positioning a milling tool (Fig. 1). Currently, this part is made by material removal in an aluminum raw and must be designed, validated, completed and delivered within $24 \mathrm{~h}$ at the latest. However, it happens that this deadline is not always respected, which causes a stopping of 
the production of parts requiring the drilling grid. Therefore the request of the manufacturer is to check if the part is achievable on an AM machine with a FDM (Fused Deposition Modeling) process in order to have a temporary drilling grid, meeting the initial specifications: having the same mechanical properties, functional specifications and surface finish constraints as the initial grid. However, the number of operating cycles can be lower since the life phase will be limited to maintain the production until the delivery of the aluminum grid. The main constraints resulting from the functional specifications of the drilling grid are:

- geometric constraints for positioning the different holes are provided by the CAD mock-up of the existing part:

- the length of the holes should be $20 \mathrm{~mm}$;

- for locking screws, the part shall withstand an axial load of $120 \mathrm{daN}$ and a radial load of $250 \mathrm{daN}$;

- for holes H1, H2 and H3, the part must withstand radial force of $37 \mathrm{daN}$;

- for holes H2, H3 and H4, the part shall withstand an axial force of 500 daN;

- the deformations and internal forces of the part should not exceed those of a part which meets these specifications, but which is made of aluminum alloy (respectively $0.0931 \mathrm{~mm}$ and $1.2910^{8} \mathrm{~N} / \mathrm{m}^{2}$ );

- the different drillings will be carried out with a dimensional tolerance of a tenth of a millimeter.

We will apply our methodology through the design of a new part which will then be compared to the characteristics of the existing part. This choice is motivated by time saving in the translation of geometric constraints from the specifications. Indeed, we will use the CAD mock-up provided by the company to ensure that the different holes are well positioned. This will also allow us to have the numerical mock-ups of elements external to the part to ensure their assembly.

\subsection{Phases of our methodology}

Our methodology is composed of three phases; this structure was intended to make more explicit the evolution of the design of the part according to two criteria that are manufacturability and optimization. The final result is of course a part manufacturable and optimized regarding the use of the material.

The first goal is to establish a primary geometry leading to a part which meets the specification, regarding physical and chemical properties, and mechanical and functional surfaces. From a purely numerical point of view, although the manufacturability of the part is not assured, it is entirely possible to stop the methodology at this level. The second phase aims to provide an optimized geometry of the primary solid to reduce the volume of material needed by the manufacturing. The issues are the manufacturing time, the amount of material consumed and the economic cost of the part. Finally, the third stage aims to ensure the manufacturability of the part. From the previous geometry obtained after the optimization, the integration of the support is envisaged and analyzed in accordance with the desired mass of the part (the importance of which may vary according life phases).

The Figure 2 presents the diagram of our methodology. This diagram will be explained and detailed in the following parts, step by step, being illustrated by the case study presented above.

This diagram is based on different components:

- boxes and purple arrows: information contained in the specifications (chemical and physical properties, functional surfaces and mechanical constraints). These data are inherently evolutionary, so the methodology must constantly adapt to any changes to the specifications, by a revalidation of the relevant steps.

- green blocks: process data that are specific to the company/the design team. These data are less likely to change during the progress of the methodology. As for the information from the specifications, these data are integrated by the methodology and help with the decision of the technical choices.

- rectangles with rounded corners: steps that directly affect the digital mock-up.

- simple rectangles: intermediate design choices, based on the data of the specifications and the process.

- solid black arrows: path of the methodology.

- dotted arrows: possible loopbacks methodology in order to validate a material, an orientation, a starting volume or geometry of the support.

\section{Phase 1: Generation of a primary solid}

\subsection{Selection of the material}

The first step of the methodology is to select materials which will used to manufacture the part. Unlike the design methodologies applied to conventional production processes, for which the choice of material is generally made after the geometric definition of the part, it is necessary to perform this step upstream. Indeed, in the context of AM, the process depends on the material, which impacts the machine used, the potential geometry and the mechanical strength of the part (which also depends on the machine) and the possibilities in terms of multi-material, support and non-removable assemblies. Although the selection of the material may be revised, (e.g., in the case where the mechanical stresses would lead to excessive deformation of the part), it is necessary to perform this step first. This choice is based on three requirements: the physical and chemical properties required by the specifications, materials to which the designer has access to, and compatibility of these materials with each other.

\subsubsection{Physical and chemical properties}

The chemical and physical properties covering all the constraints related to the material properties: dissolution in a solvent, elasticity, magnetism, thermal or electrical conductivity and so on. At this level of the methodology, no geometry part having been defined, these constraints are independent of the mechanical constraints and those 


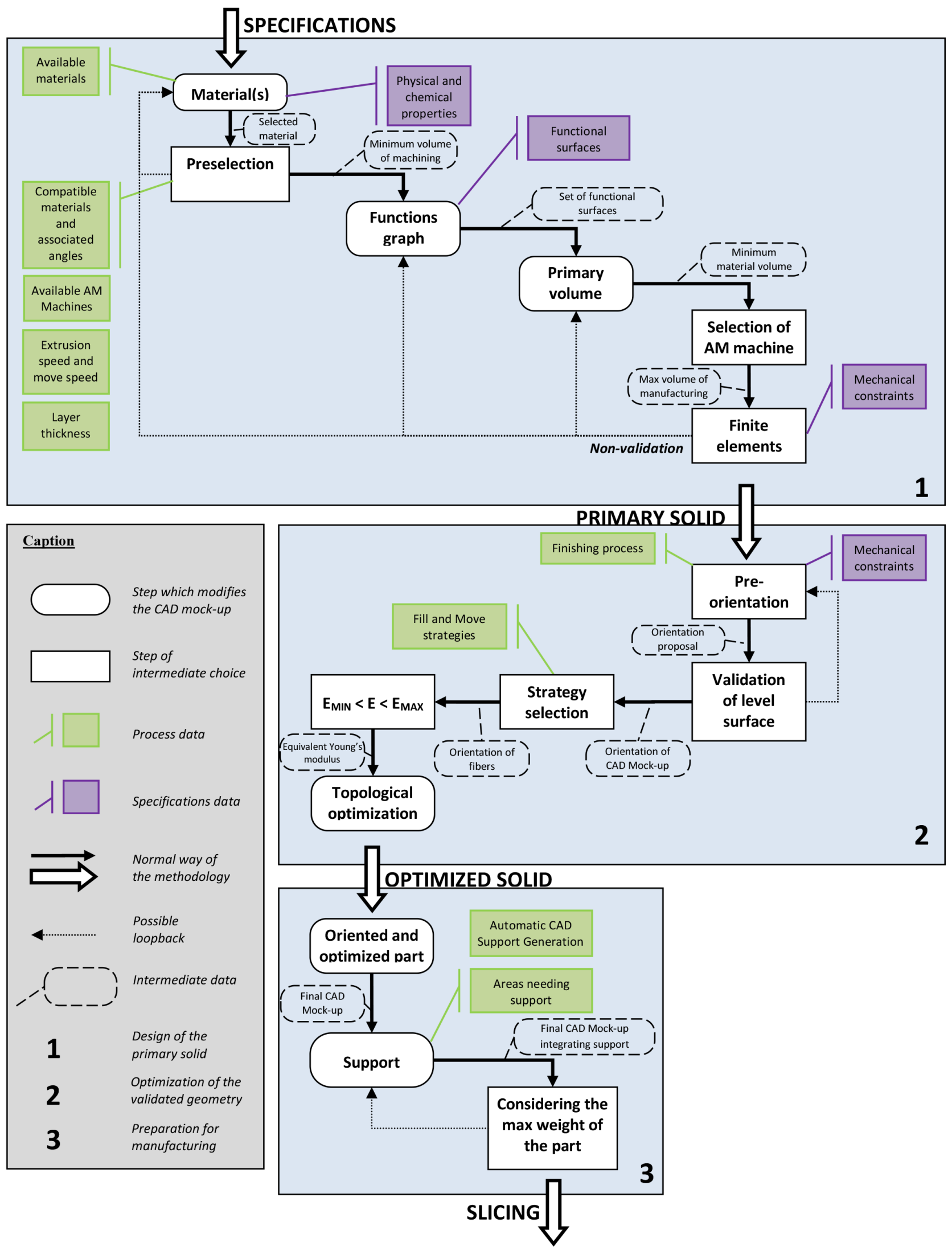

Fig. 2. Diagram of the design methodology. 
related to the functional surfaces. The purpose of this step is only to verify that the physical and chemical constraints allow the selection of at least one type of existing material. There are three scenarios for the selection of the material, according to the constraints applied to the part:

- no constraint or only one constraint: use of a single material.

- a property is desired on a specific area of the part: multi-material fabrication. This possibility is subtended by the compatibility of the materials during the manufacturing phase.

- at least two physical or chemical constraints which require at least two incompatible materials: cutting the piece in subassemblies.

Although we anticipate the three possible cases, we treat here parts composed of a single material, since current finite element analysis tools address only parts composed of a homogeneous isotropic material or heterogeneous orthotropic but not heterogeneous anisotropic. Our methodology will be enriched as early as the tools will enable an analysis of multi-material parts.

Note that we will restrict the methodology to the materials that can be implemented on an FDM machine type, as this is the type of process both more widespread and with the highest specificities: the inclusion of a support case and the possibility of producing multi-material parts, unlike the methods of stereolithography or powder bed.

\subsubsection{Compatible materials and possible materials}

Possible materials refer to all materials available to the designer, depending on the compatible available machines. Compatible materials refer to any materials that can be simultaneously used during a same manufacturing phase for a same material deposition process (FDM CLAD, PolyJet ...). Material compatibility is primarily imposed by their glass transition temperature.

\subsubsection{Material selection}

Regarding these constraints, several materials may be eligible. In this case, the choice will be based on the constraints of the company policy: material cost, material recyclability, ease of supply, machine availability and so on. Several selection methods could be used as for instance weighted average method AHP and ELECTRE 1 method [24]. It is therefore difficult to advocate a single method. If no selection method can discriminate a material, it will be possible, at first, to regard the cost of the material and possibly revise this choice after a step of finite element analysis.

\subsubsection{Application to the case study}

For the studied part, no physical or chemical property is imposed by the specifications: our methodology therefore indicates that a single material can be selected. To reduce the manufacturing cost, we choose the ABS. This choice may be revised during the finite element analysis.

\subsection{Pre-selection of the machines}

The pre-selection of the machines consists of successive phases of elimination, the first being the elimination of incompatible machines with the selected material. At this stage of the methodology, integration support cannot be addressed as the digital mock-up of the part does not yet exist. However, in the case of FDM method, we anticipate the presence of soluble support (it appears that the resulting model of a geometry optimization is often composed of very complex shapes for which the manual removal of the support cannot be guaranteed). Therefore, this methodology assumes that the support may be made of a second soluble material compatible with the material of the part. This constraint makes it possible to narrow the first list of machines. If the support has to be carried out in a nonsoluble material, our methodology provides no indication to the designer regarding the possibility of its extraction. It may nevertheless change, in accordance with the specifications, the geometry of the part to limit the presence of the support.

At the end of this stage, the outputs are the material used for the part as well as a list of machines ideally usable. We consider the general case of an optimized part with a complex geometry involving the presence of support.

\subsubsection{Application to the case study}

We have four machines: MakerBot Replicator Dual Extrude, MakerBot Replicator 2X, Spiderbot and Zcorp z510. First, by applying our methodology, we therefore exclude the Zcorp z510 as it works with powder bed disposition. Secondly, we exclude Spiderbot machine because it has only a single nozzle (it will not allow to achieve a possible support of a different material. The short list is then composed of the two Makerbot machines.

\subsection{Graph of functions}

The third step of the methodology is to establish the graph of functions. The designer will create a digital mock-up of the piece by integrating the constraints and functional surfaces from the specifications directly in a CAD file. The term graph corresponds to the representation of the product in entities and links between these different entities. These entities may contain one or more functional surfaces. This graph representation takes into account the geometrical and dimensional specifications connecting the different entities.

\subsubsection{Functional surfaces}

Functional surfaces corresponding to all the contact surfaces of the part. They correspond to the constraints of the specifications, which can be represented by a geometric shape. These surfaces should also incorporate, if possible, notions of surface finish and geometric tolerance. It is necessary to differentiate the functional surfaces of design, where the designer has complete freedom regarding the geometry (related to aesthetic and ergonomic choices) and functional surfaces of constraint for which 


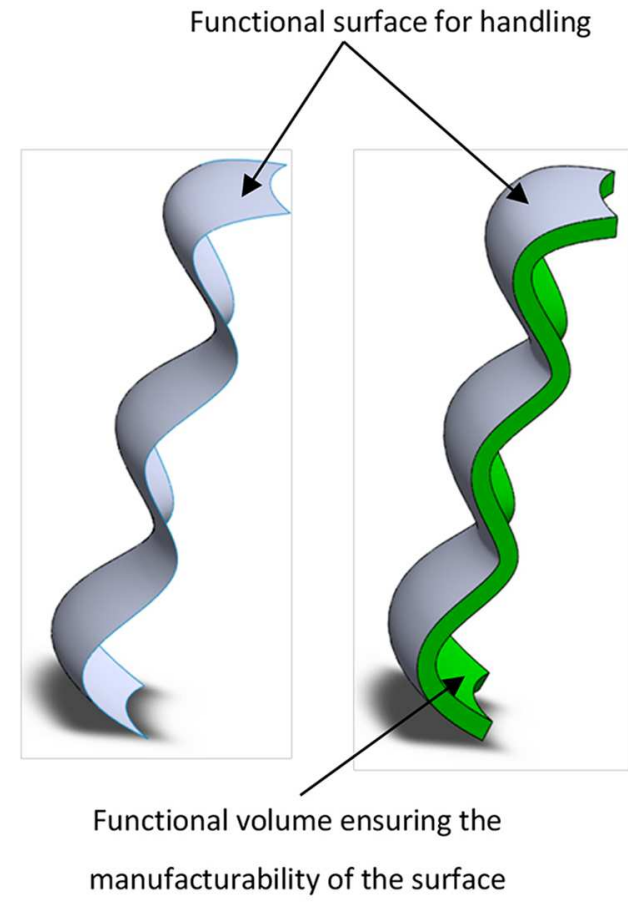

Functional surface for screw

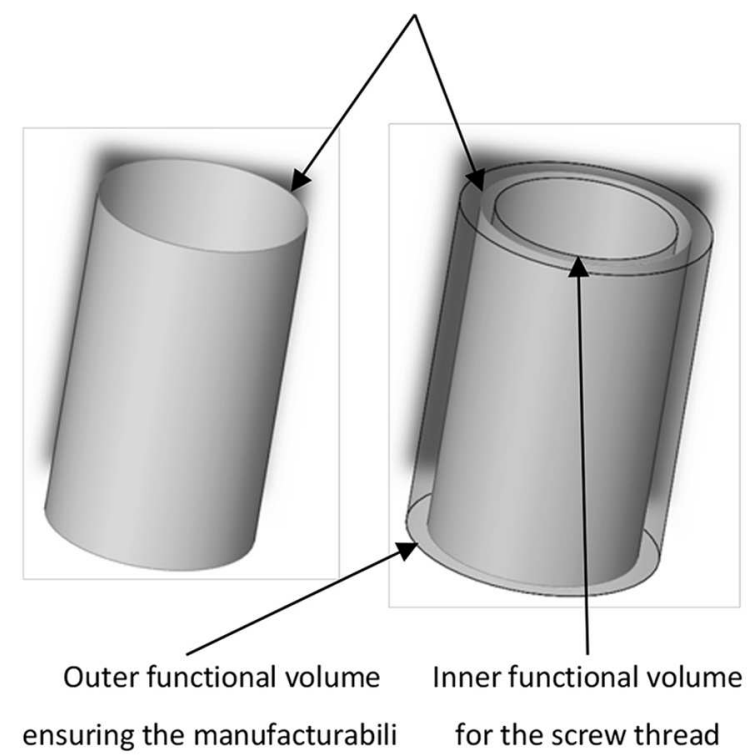

of the surface

Fig. 3. Functional volume of constrainty.

the designer is forced to follow a predefined geometry. These surfaces are stemmed from the surfaces of existing elements to which the part to design must adapt (e.g., screws, nuts, bearings and so on or another part of the graph). In all cases, the surface to be considered is whether existing as digital mock-up or convertible in a CAD mock-up.

For each functional surface of constraint, it is necessary to integrate a functional volume of constraint reflecting the need to have an increased thickness, to allow the generation of the surface and, when appropriate, the establishment of the outer element (Fig. 3). However, there is no recommendation about the exact thickness of this excess thickness as it depends on the material and the orientation of the part.

In the case of design surfaces, the designer does not necessarily have knowledge of the shape of the functional surface. For a given constraint, there may be a whole range of functional surfaces. One goal of our methodology is therefore to dynamically change the geometry of these surfaces during the design while maintaining the already integrated elements. Moreover, for a given volume of material, any change in the digital mock-up only impacts marginally the final cost of the part since the planned machine remains the same.

Finally, it is necessary to include the expected surface finish, the geometric tolerance, the manufacturing direction and the volume of contact. The latter is a volume supported on the functional surface and directed towards the outside and which should be left blank during the design phase to allow the integration of external elements after manufacturing of the part.

\subsubsection{Generation of the graph of functions}

To set the graph of functions, the designer has to give all the functional surfaces required for the part in a CAD software. The creation of the graph, as shown in Figure 4, is described thereafter.

Each surface stemmed from the specifications is first recorded in a separate part file (Fig. 4.1). This allows the design team to modify, if necessary, each surface independently. It also allows to form a functional surfaces database that can be reused for another design. In addition, these surfaces can be used both for interior or exterior contact surfaces as they have opposite normal in the CAD software.

Each constraint from the specifications, not translatable in terms of surface, is also recorded in a part file (presence of a specific material, surface finish etc.). At the beginning of the design, a constraint is represented by a sphere in the CAD software. This representation is intended to give the designer the presence of an untreated constraint. These constraints do not necessarily impact the form or the final volume of the part (mechanical strength, conductance etc). As the design advances, these constraints will be associated with a surface or volume of the part.

Once all surfaces and all necessary constraints integrated into the CAD software, the files are then integrated in an assembly file that allows the independent update of the functional surfaces (Fig. 4.2). The designer can then spatially organize the different surfaces and add any positioning constraints related to dimensional tolerances (Figs. 4.3 and 4.4). 


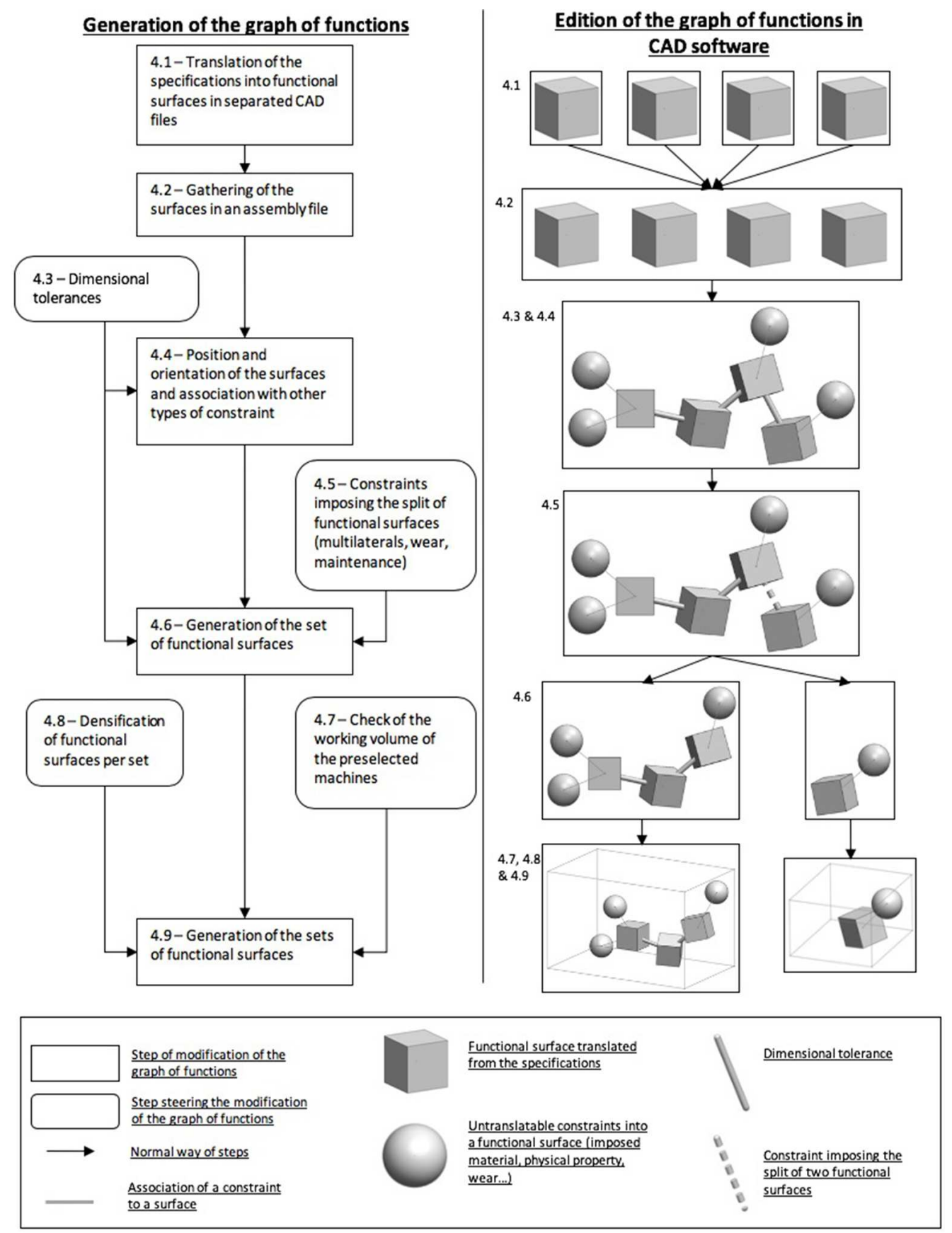

Fig. 4. Generation of the graph of functions. On the left, the different steps of the generation method, and on the right, an illustration of these steps by editing the function graph using the CAD software.

Even if the part is achievable in a single entity, it may be necessary to separate some surfaces (Fig. 4.5) according to the constraints associated with the specifications (e.g., wearing surface). In this case, the functional surfaces are grouped in different sets, each set leading to the realization of a different physical part. Every feature of the specifications is represented by a single surface having surface finish and geometric tolerances. Geometric tolerances imposed by the specifications are linked in one set to determine the most suitable machine based on the volume of each set (Fig. 4.7). If a set formed in this manner could not be contained in any workspace of preselected machines, it is necessary to check if some geometric tolerances can still be ensured despite the separation of the surfaces in several sets (Fig. 4.9).

The decomposition of the graph into different sets is based primarily on the constraints given by the specifications and is performed in different steps (Fig. 4.6). The first step is based, as indicated above, on the presence of demountable surfaces. The second step is based on the assumption that AM allows the realization of fixed volumes having relative movements during the use of the 


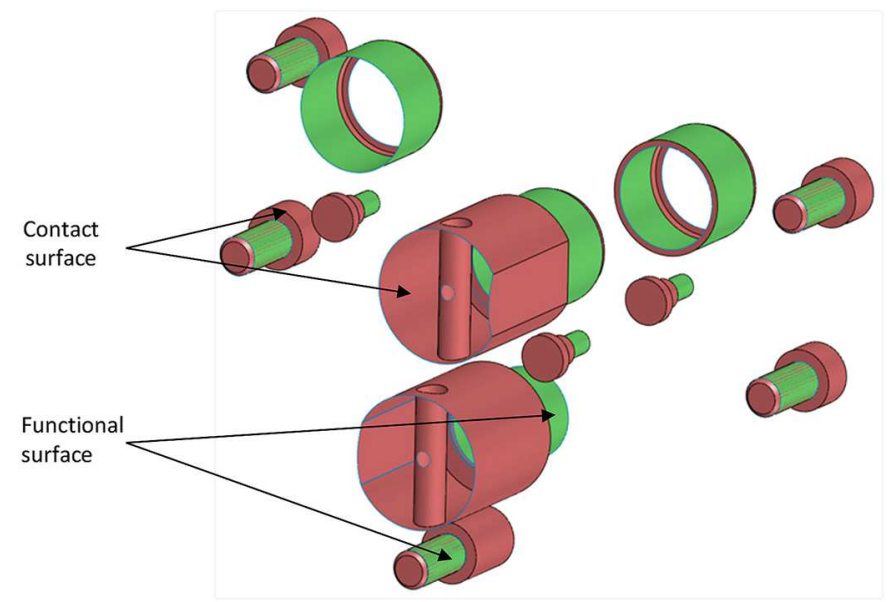

Fig. 5. Graph of functions of the case study, final representation.

part. Secondly, it will be necessary to identify which bodies against each other: each body will generate a set. The third step of decomposition is based on the assumption that AM is not limited either by the complexity of the pieces, either by producing multi-material parts. This step is most important since the aim is to centralize a maximum of constraints and functional surfaces on the same set unlike most design methodologies adapted to standard manufacturing processes (Fig. 4.8).

Ideally, each part to be produced consists of a single set. If a part is made of at least two sets, functional joint surfaces are integrated in the graph of links. The principle of assembly (e.g., welding, gluing, videos, riveting, bolting and so on) depends on the material chosen above, and on the functional surfaces. However, since AM does not impose any limitation on the geometry of the parts, we will assume that any part is manufacturable, regardless of the number of sets.

\subsubsection{Application to the case study}

In the framework of our work, we use SolidWorks as CAD software. We begin to generate the graph of functions from the given surface constraints: the part needs to incorporate thirteen borings lying between two parallel planes distant by $20 \mathrm{~mm}$ and needs to receive an existing part (drilling tool, bearing, fast positioning screw or pin). Each of these pieces has a have a plane-parallel contact surface on the drilling grid, which means that each drilling has a contact surface and an associated contact volume.

Since the specifications also provided an existing CAD mock-up (Fig. 1), we must also impose positioning constraints at this stage. Regarding the case study, it is possible to connect all the functional surfaces same set. Figure 5 presents a representation, on which only surfaces are represented: green areas correspond to functional surfaces and red areas correspond to contact surfaces.

After the selection of the machine, if the set was not encompassed by the work volume, it would be necessary to loop back on this step to separate the set into sub-sets.

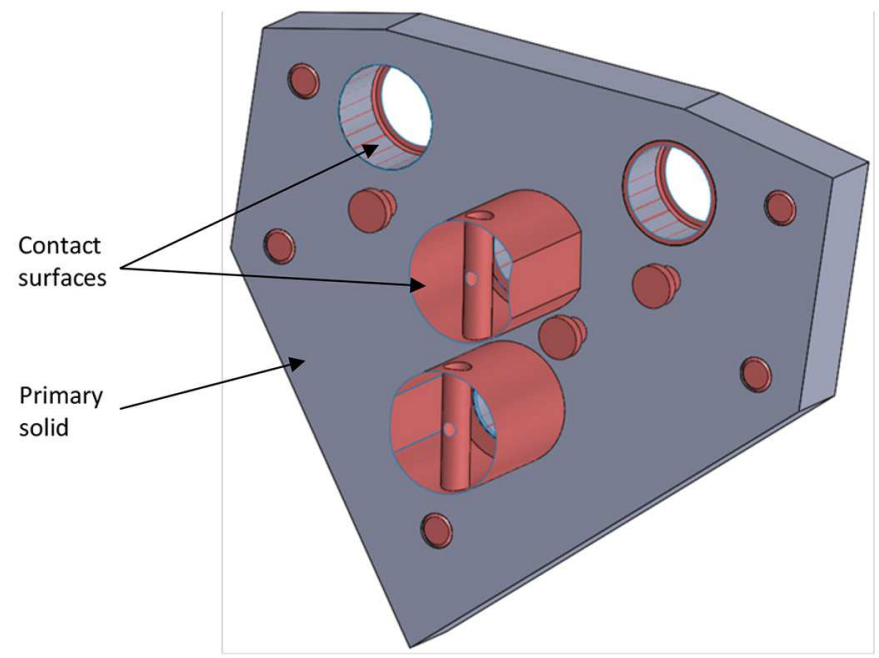

Fig. 6. Primary solid of the case study.

\subsection{Definition of the primary solid}

From the graph of functions, the designer can establish a primary solid for each set, which consists of a single solid body, which is a necessary condition for the steps of analysis and optimization that come later in the methodology. It must also include solid material thicknesses that meet the constraints of minimum thickness of material that the machine may deposit.

At this stage of the design, it is assumed that all the functional surfaces and design constraints are placed correctly. However, none of these surfaces is necessarily connected to the other and only the functional volumes of constraints were integrated. Based on the functional surfaces, the designer starts by generating the functional volumes that take into account the minimum amount of material required for the manufacture of these surfaces and, if necessary, to the integration of external elements.

A first draft of the solid is obtained by connecting the functional volumes two by two after a Delaunay triangulation to obtain the smallest volume encompassing the functional volumes. In addition, this volume presents the smallest possible mesh for finite element analysis. At this point of the methodology, the designer has a unique solid body per set, respecting the functional surfaces and chemical and physical properties of the specifications.

\subsubsection{Application to the case study}

We generate the primary solid based on the functional surfaces. For functional volumes, regarding business knowledge, we will take a minimum material thickness of $5 \mathrm{~mm}$. This allows us to obtain a first set of functional volumes. Delaunay triangulation on these first volumes is relatively simple because all the functional surfaces are parallel cylinders bounded by two parallel planes and perpendicular to the axes of the cylinders. The generated primary solid respects both the functional surfaces, functional volumes as well as the contact surfaces (Fig. 6). 


\subsection{Selection of the machine}

From the primary solid and the shortlist of suitable machines, it is now possible to determine the machine that will be used. This choice is based on the following constraints: working volume, extrusion speed and displacement, resolution and accuracy. The resolution corresponds to the height of each layer of material. The accuracy of the machine corresponds to the various tolerances that can be obtained according to the various axes of the machine. This parameter varies with several controllable parameters as filing strategy, the orientation of the part or the position of the part on the tray, but also the material used on the machine [25]. The accuracy also depends on fixed parameters such as machine architecture ant its materials. The resolution is very important because it will determine the orientation of the part in the design to respect the surface finish imposed by the specifications. The finest surface finish is always obtained in the following three cases:

- in the manufacturing direction;

- in an inclination close to the manufacturing direction;

- perpendicular to the manufacturing direction.

By contrast, more the inclination of the surface of the part moves away from the perpendicular to the machine direction, more the \stair phenomenon" becomes visible. Although a surface treatment is sometimes possible, it is always better to limit the number of steps required to obtain a functional part. In addition, the generated surfaces may not be accessible for a possible finishing operation. We must find a compromise between the constraints of surface finish, the orientation of the part, and the accuracy and the resolution of the machine.

\subsubsection{Application to the case study}

The resolution $(0.1 \mathrm{~mm})$ and the nozzle positioning accuracy $(2.5 \mu \mathrm{m}$ in the $x$ and $y$ axes, $11 \mu \mathrm{m}$ in the $z$ axis $)$ are equivalent for the two machines of the shortlist. However, the MakerBot Replicator 2X only has a superior workspace on each dimension of the bounding volume of our primary solid, which determines the choice of this machine.

\subsection{Finite element analysis}

To continue the design, it is necessary to ensure that each set is validating the mechanical constraints stemmed from the specifications. Otherwise, our methodology would result in a deadlock in the topology optimization step. The realization of a part layer by layer in AM requires an anisotropic structure of the material. The orientation of the part thus determines its mechanical strength. The strategy is to conduct a finite element analysis in the worst case, so as to anticipate all possible orientations. We will set the Young's modulus of the material to its minimum value for this step; this minimum value is obtained when the force is parallel to the manufacturing direction.
If the part can be manufactured in a single volume, the preparation of the finite element analysis is to apply all efforts (stemmed from the specifications) on a single part. The result proposed by the analysis software allows to know immediately if the part meets the specifications regarding its mechanical strength. If the part is divided into sets, the designer should perform a finite element analysis on the overall part. Then, for each set, the software integrates calculated efforts at the interface between the two contact surfaces, to determine whether the resulting part conforms to specifications despite the areas weakened by assembling sets. In these two cases, if the finite element analysis indicates that the piece is resistant to mechanical constraints of the specifications, the primary solid is validated and the designer can switch to the step of the orientation of the part.

If the analysis indicates that this part has some fracture areas, the designer has to apply a specific procedure. First, the designer can reinforce the part on the areas indicated by the finite element analysis. Indeed, the volume generated via a Delaunay triangulation is not necessarily optimized regarding the forces. After the modification, the designer must perform an additional finite element analysis to see if the primary solid satisfies the constraints of the specifications. If this is the case, the solid is validated and the designer goes to the next step, if not, the designer loops back to the reinforcement of the part as the constraints of the specifications and the workspace of the machine allow him. Otherwise, the designer has a second option.

The second option is to slightly raise the Young's modulus without impacting the surface finish of the functional surfaces. Analyzing the orientation of the stresses and of the functional surfaces, the designer orients the workpiece so as to position the effort as perpendicular as possible to the manufacturing direction while validating the surface finish of the functional surfaces. If there is an orientation of the part which meets all these conditions, then the designer can increase the value of Young's modulus to run a new finite element analysis on the part. If the part meets the mechanical constraints, the designer goes to the next step of the methodology. Otherwise it is possible to repeat this second option 2 or to consider the third option.

The third option should be considered a last resort: it consists in the changing of the material of the part and imposes to start all the methodology again. Indeed, a new material potentially implies a new AM machine, and by domino effect a new manufacturing volume which may imply a new division of the sets which can impose new primary. To know when to consider this option, a good indicator is the maximum weight of the part.

Since the primary solid will go through a topological optimization to reduce the amount of material, it is possible to consider options 2 and 3 without changing the geometry of the previous primary volume.

\subsubsection{Application to the case study}

We consider the worst case: ABS is printed perpendicular to the directions of efforts. The efforts stemmed from the specifications have been placed on the initial geometry of 

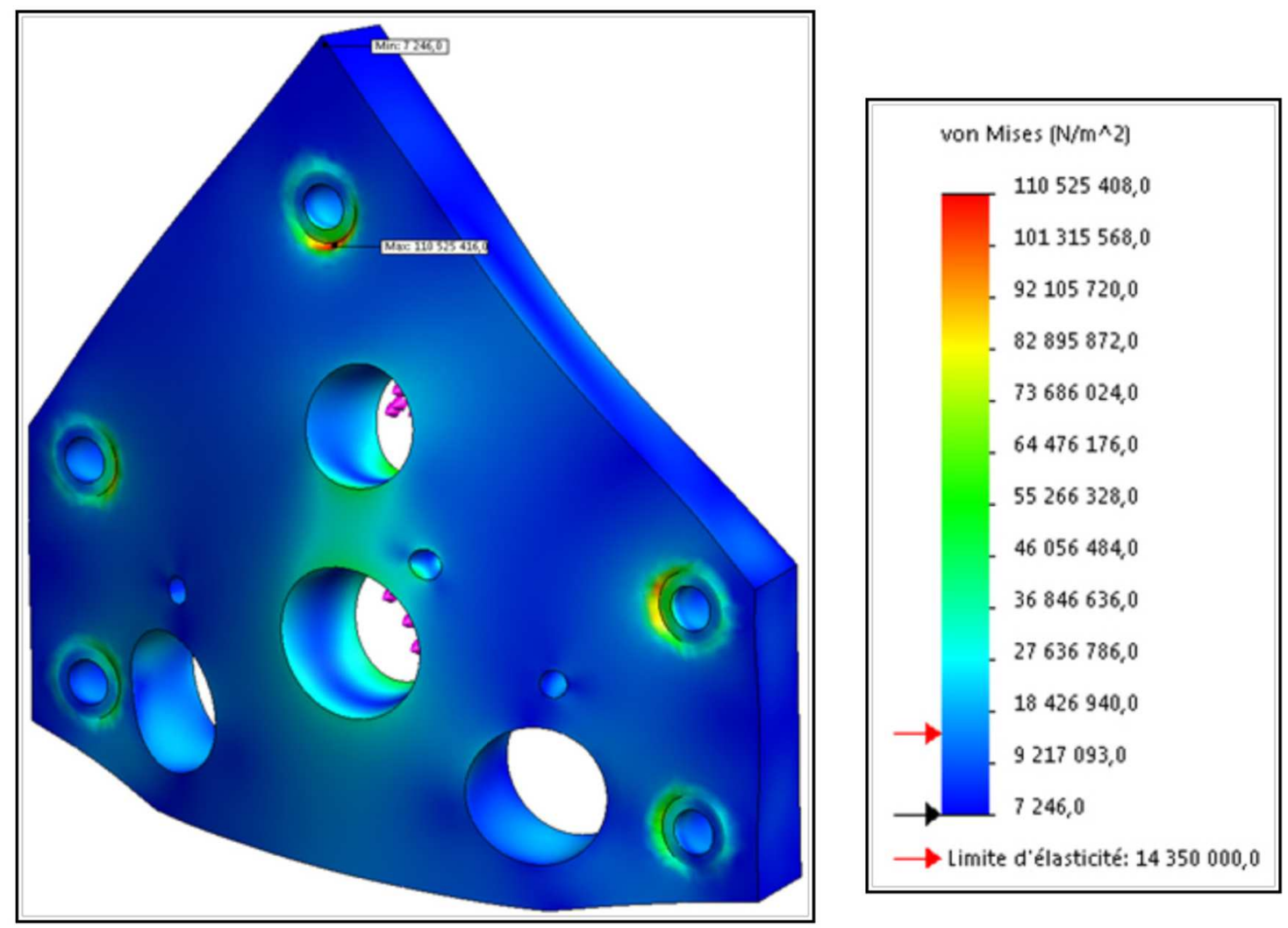

Fig. 7. Results of the finite elements analysis for the initial grid in ABS with minimum E.

the primary solid. The result of the first finite element analysis (FEA) is presented on Figure 7. We find that the forces applied lead to a breaking of the part. We choose to thicken the part of $20 \mathrm{~mm}$ from the tool side, which is the limit to respect contact volumes and to perform a second FEA. As the result is not valid, we thicken the part of $3 \mathrm{~mm}$ on the area concerned, while respecting the minimum distance between the drilling grid and the part.

Despite the increase of the volume of material, the part still does not meet the specifications. We must therefore consider modifying the Young's modulus. In order to respect the surface condition of the drilling and to avoid "staircase effects", it would be preferable to make them parallel to the manufacturing direction, which is also the ideal orientation for resistance to shear stresses of the part. We are in the ideal case where the Young's modulus is maximum, which allows us to realize a fourth FEA. Nevertheless, the piece still does not meet the specifications. It is therefore necessary to consider a change in the material, and to replace ABS by ULTEM (proposed by the specifications). For this configuration (Fig. 8), the part meets the specifications.

At this point in the methodology, the most important part of the design work is realized: the resulting part respects the specifications particularly in terms of mechanical strength, physical and chemical properties and for which shapes allow the assembly with all the outside elements. In addition, we have the guarantee that this part is manufacturable on at least one AM machine.

\section{Optimization of the primary solid}

\subsection{Pre-orientation of the part}

The aim of this step is to find a compromise between maximizing the surface finish of functional surfaces and maximizing the strength of the piece depending on the orientation of efforts, to determine the ideal manufacturing direction, if this direction has not been fully defined in the FEA. This orientation is carried out automatically under SolidWorks using a multi-objective optimization controlled by the surface finish in the worst case. Currently, this indicator is not yet implemented.

If the indicator is lower than or equal to the specifications of the surface finish, the functional surfaces will have directly a satisfactory surface finish during the manufacturing phase. Otherwise, it means that a finishing operation is envisaged after manufacturing of the part. The value of the indicator depends on the ease of realization of a finishing operation for the selected material.

An abacus, provided to the designer, shows the maximum values of surface finish that can be obtained depending on the material and the accessibility of the surfaces. Once the value selected, optimization provides one or more directions of the part allowing to obtain the surface finish and the best mechanical strength. If no proposal can satisfy both the mechanical and surface finish constraints, it is necessary to revise, if possible, the 

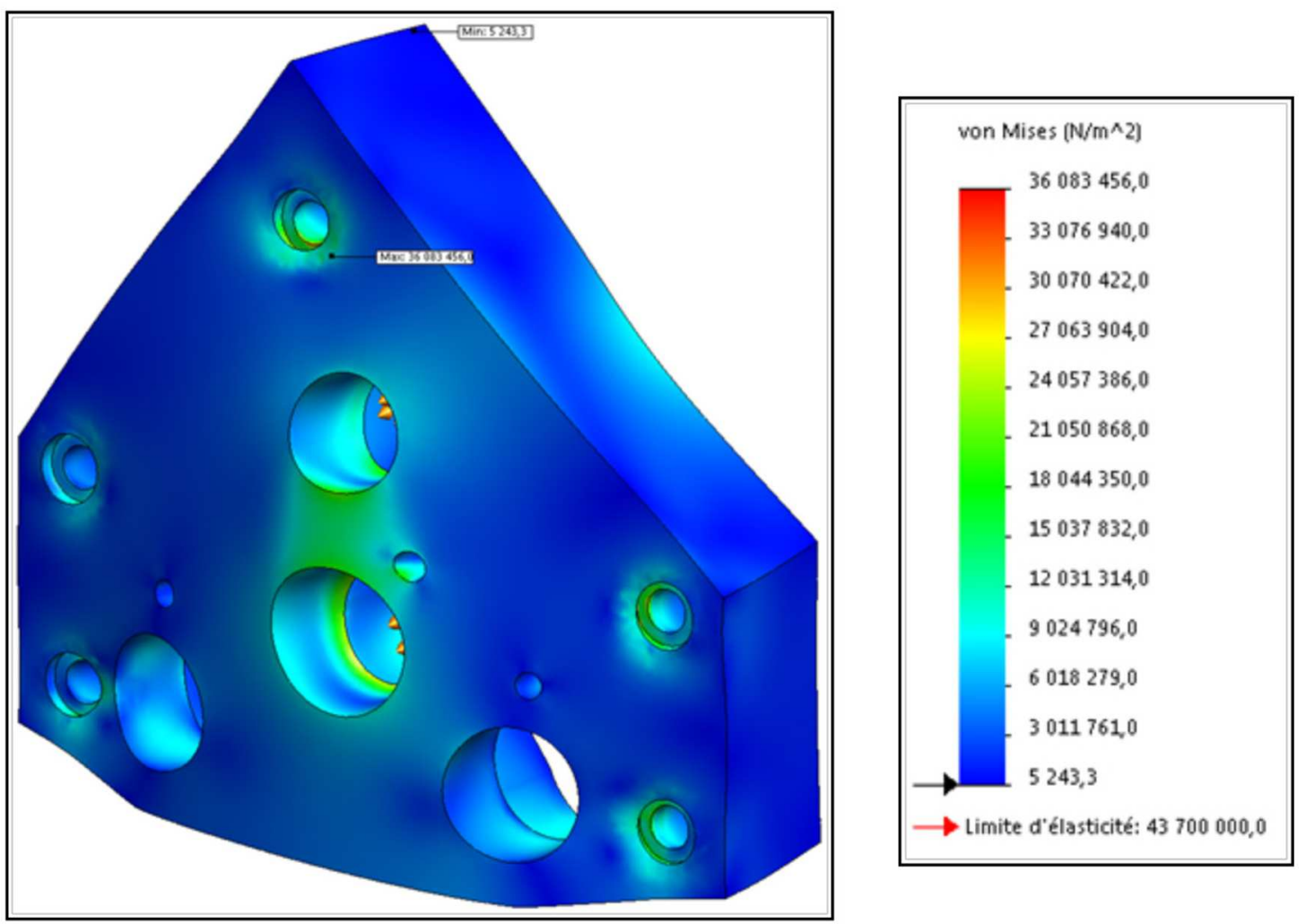

Fig. 8. Results of the finite elements analysis for the final grid in ULTEM with maximum E.

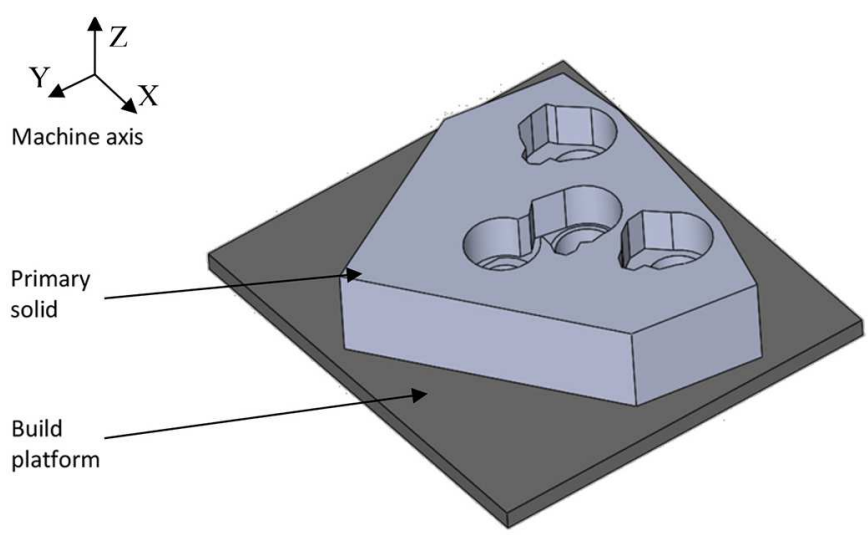

Fig. 9. Oriented drilling grid.

constitution of the different sets, in order to limit the number of constraints for each part. If the geometry of the solid cannot be changed, the designer should consider changing the material to gain more freedom in the orientation.

\subsubsection{Application to the case study}

In our case, the part was directly properly oriented at the FEA step: efforts are oriented to best comply with the manufacturing direction ( $z$ axis) and the surface finish of each functional surface is in accordance with the specifications (Fig. 9).

\subsection{Strategy selection}

The designer must select a final orientation (single rotation about the $z$ axis depends on the volume available machine and the shape of the part) and a strategy of manufacturing, based on three main criteria: the moving strategy, the filling strategy, and the fiber orientation.

\subsubsection{Moving and filling strategy}

The strategy is the path followed by the print head and can be divided into three main criteria: the thickness of the inner and outer surfaces that influence the thickness of the surface of the part, and the internal filling which a variable density of permanent support used to stiffen the part.

According to the forces applied to the part, it is advantageous to orient the fibers in order to adjust the strength of the part. Once the strategy is determined, it will be necessary to obtain a model of the mechanical behavior of the part to estimate an equivalent Young's modulus for the topological optimization phase.

\subsubsection{Selected strategy}

At this stage of the methodology, the part satisfies all mechanical constraints. The method involves orienting the fibers parallel to the largest traction effort to maximize the strength of the part. However, when the material solidifies, it tends to contract [26,27] and, in the case of FDM, 


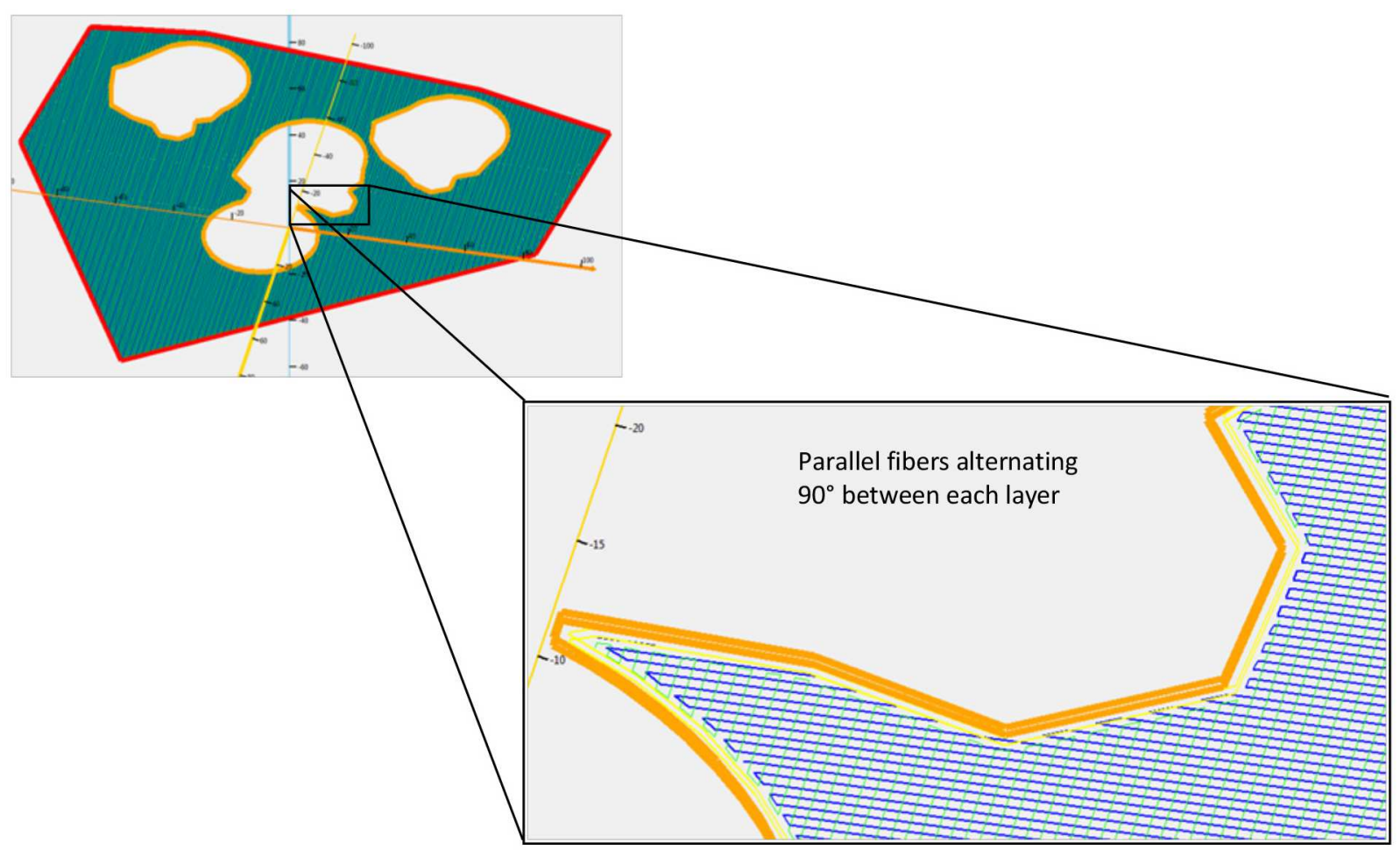

Fig. 10. Extract of the machining strategy of the case study.

it induces a warping effect. To prevent this, it is advisable to limit the length of the fibers.

Again, the software helps the designer indicating the equivalent Young's modulus resulting from the fiber orientation and areas where the risk of warping is high. However, it is impossible for the software to perform a reliable prescription given that the part has not been topologically optimized. This step will therefore fall within the expertise of the designer who have to choose the most appropriate manufacturing strategy.

\subsubsection{Application to the case study}

In our case, no traction effort is applied to the part in the manufacturing plane. In addition, the equivalent Young's modulus equivalent is already maximum with the orientation of the part and of the efforts. The strategy which limits the embossing effects is presented in Figure 10.

\subsection{Young's modulus}

The Young's modulus is estimated by the CAD software according to the selected material and the previously selected strategy. The Young's modulus may vary between the values Emax, which is the Young's modulus for an isotropic, homogeneous and uniform material, and Emin corresponding to the Young's modulus for the same material but made in AM with the worst conditions.

\subsection{Topological optimization}

The aim of this optimization is to minimize the required volume of material, the manufacturing time and therefore the cost of the part. Commonly, the designer uses CAD software module to perform a topological optimization on the primary solid. For our methodology we use PareTOWorks plugin. As this plugin only deals with stl files, the designer can specify the surfaces that should not be affected by optimization, to maintain the functional surfaces. The result of this optimization is a second stl file whose resolution can be assigned manually by the designer.

\subsubsection{Application to the case study}

Using the specifications and the primary solid, we can perform a topological optimization step. We obtain an optimized and oriented part (Fig. 11); areas which does not support any effort have been reduced in order to limit the amount of material required to manufacture the part.

\section{Preparation of the part to the manufacturing}

At this step, the designer obtains a warped part without predefined geometry (apart from the geometry of the functional surfaces) to which he has to check the presence of support during the manufacturing phase.

\subsection{Minimal angle of support}

Each material is associated with a minimum angle of support which corresponds to the maximum acceptable banking angle before the collapse of the material. For the FDM process, the value of this angle is $45^{\circ}$. Given the 


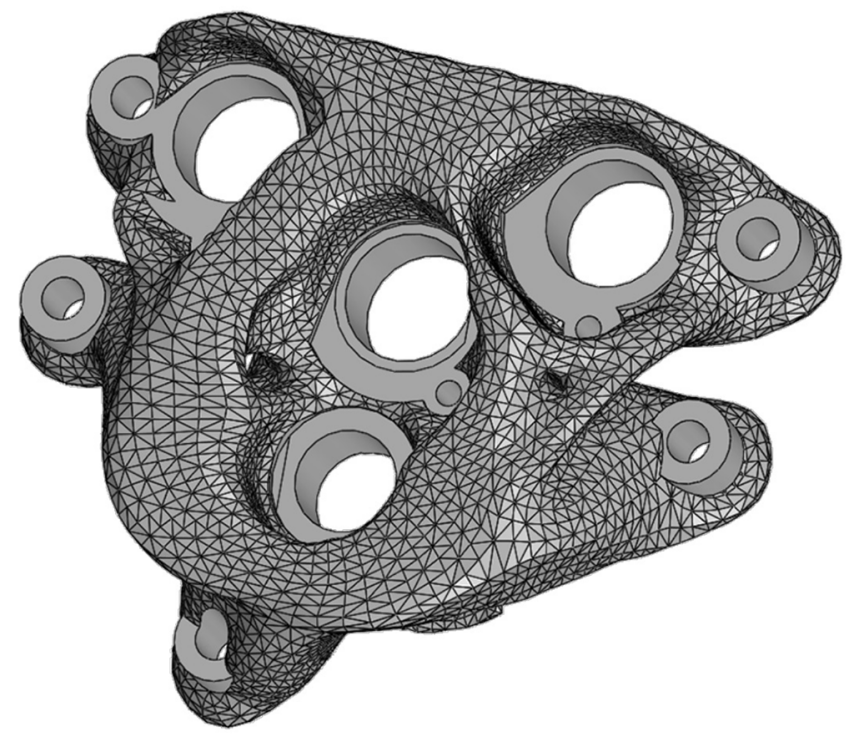

Fig. 11. Oriented and optimized drilling grid.

orientation of the part in the machine space, if at any point of the surface of the part, the angle between the tangent at this point and the printing direction is smaller than $45^{\circ}$, the part can be fully realized without support. Once part is oriented, it may be appropriate, if possible, to modify its surface by adding material in order to decrease the value of the minimum angle of support below $45^{\circ}$ to minimize the volume of the support (Fig. 12).

\subsubsection{Areas requiring support}

Currently, there are various software allowing automatic generation of support based on the geometry and the orientation of the part [28]. However, in the case of FDM process, the generation of the support is not optimally performed and has two major drawbacks. First, the volume of the support is the orthogonal projection of the surface to be supported. Second, the extraction of the support is not taken into account, which can lead to an impossible extraction for some cavities. Therefore, we assume that the areas requiring support must be given to the designer so that it can create and correctly position the supports directly during the edition of the digital mock-up. To determine these areas, see [29].

\subsubsection{Editing of the part}

Once the presence of zones which require support are identified, the designer can modify the surfaces of the part. By adding material, he can change the inclination of the surfaces to attain the minimum angle of support. The part will always respect the mechanical specifications because it was previously optimized.

If the priority is given to minimizing the weight of the part (e.g., for an aeronautic part), it is preferable to preserve the mock-up derived from the topological optimization and to integrate the support where it is needed. Similarly, the size of the series can impact the presence of the support according to the cost of the material and the manufacturing time. The selection criteria are the final weight, the cost, the manufacturing time and the finishing time of the part.

\subsubsection{Application to the case study}

As the part is relatively complex to study, we chose to restrict the study to a section of the part. The area studied in the optimized drilling grid is shown in Figure 13.

\subsection{Support}

The final step of the methodology is to incorporate, if necessary, the supports to the part. As for the part, our methodology is designed to reduce material and times costs. For this step, which is specific to the FDM process in the framework of our work, the designer will rely on an analysis and support generation software taking as input the minimum angle of the support, the minimum angle for supporting the support and materials compatible with the material of the part.

\subsubsection{Automated CAD support generation method}

This method generates destructible support with a predefined geometry designed to minimize the volume of material used for the support. The details of this method are presented in [29]. In the previous step, we determined the areas where the support is required. In the same way that the CAD software can generate the surfaces where support is needed, it is possible to generate the volumes of the supports. Using the minimum angle of support, the generated supports can be porous and thus have a large contact area with the solvent. This method thus allows to save time in the support generation and cleaning.

\subsubsection{End of the methodology}

Once the digital mock-up of the support is recorded in a part file, it remains to perform the slicing operation and then merge the generated code with part one to manufacture the part.

\subsubsection{Application to the case study}

The final piece (with its support) is presented in Figure 14.

\section{Results}

The methodology we have described presents a paradigm shift compared to conventional design methodologies. Indeed, by using the graph of links, it is possible to start the detailed design of the part without validating any architectural design. This allows a greater design flexibility.

In contrast to current methodologies where the geometry of the part overrides the constraints of the specifications, the AM process allow to freely consider these constraints from the beginning of the methodology. The first step is to select the material (one or more) of the part. The designer can then freely define the geometry of the 


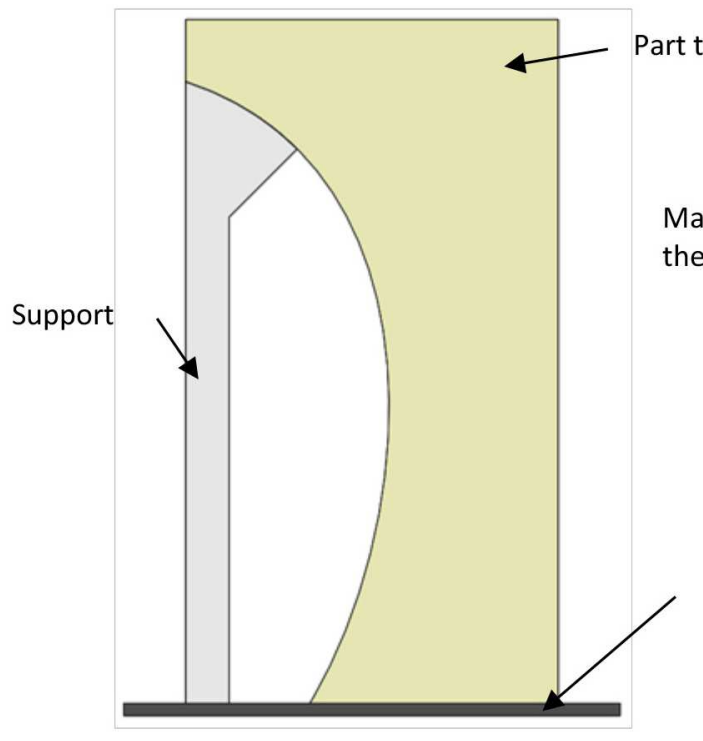

Geometry of the part imposing support

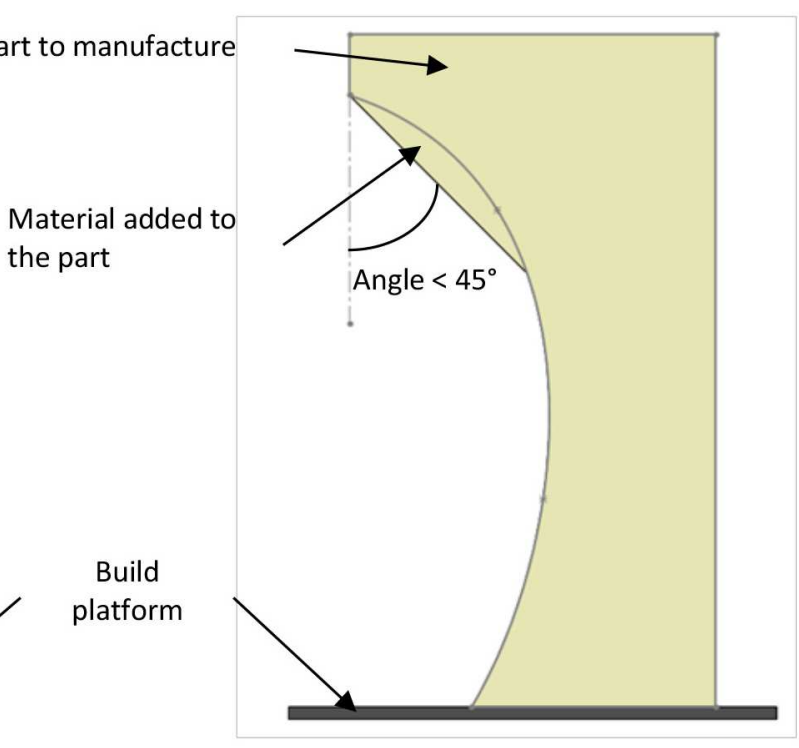

Avoiding the presence of support by the modification of the geometry of the part

Fig. 12. Decrease of the support volume by the modification of the geometry of part according to $\alpha$.

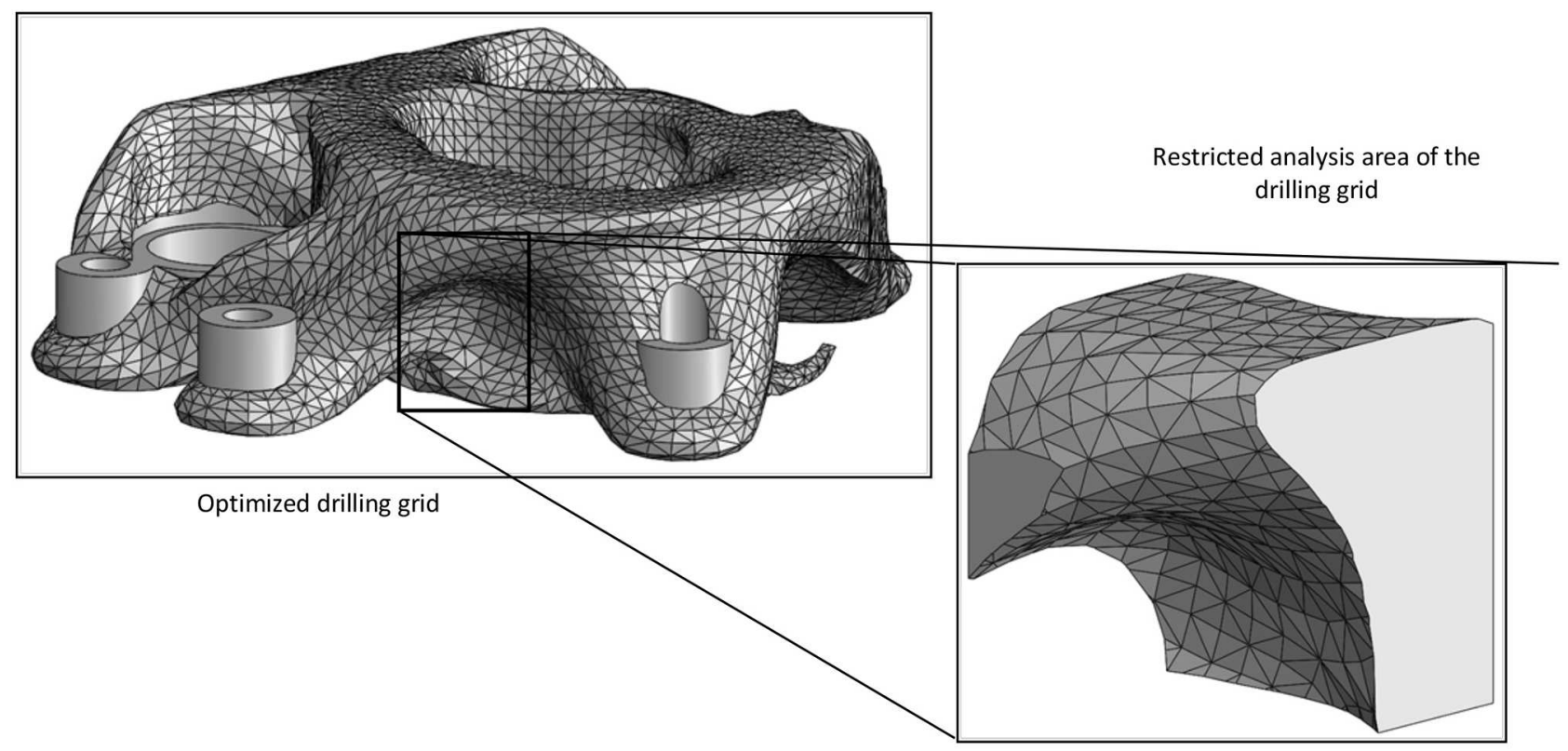

Fig. 13. Areas needing support.

part. Of course, this geometry is mechanically validated using a finite element analysis.

Our methodology brings new elements over existing methodologies. Our methodology propose provides an analytical tool, the graph of functions, which starts directly from specifications. This graph translates the constraints of the specifications directly into functional surfaces. Beyond the contribution of this graph, we also propose a method for editing this one. Our work also focused on the support, with the proposal of a new and original method of optimization (volume and cleaning). We propose to realize the generation and the integration of the support directly into the CAD software. This technique has the advantage of preserving the continuity of the digital chain. In addition, the designer's work is facilitated by the manipulation of a single tool. Finally, the three parts of our methodology can be applied independently. It is possible to focus only on obtaining of a functional part (part 1) without considering optimization or support. It is also possible to generate an optimized support (part 3) on any existing part. 


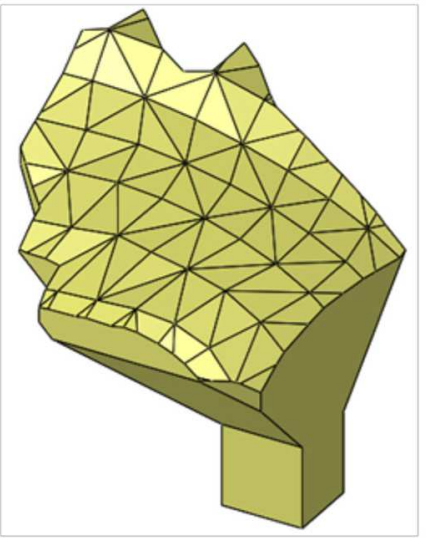

Left support, view from the surface to support

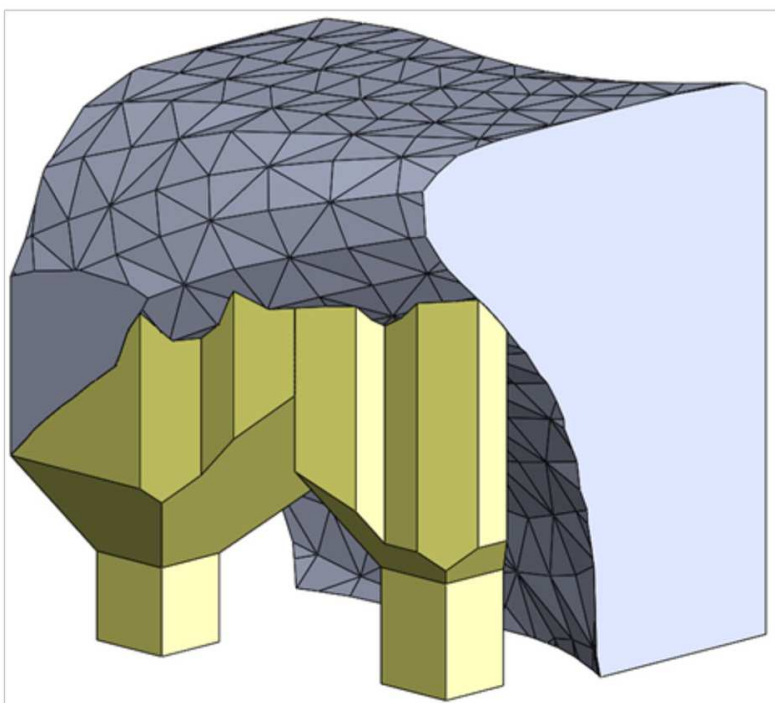

Drilling grid integrating support

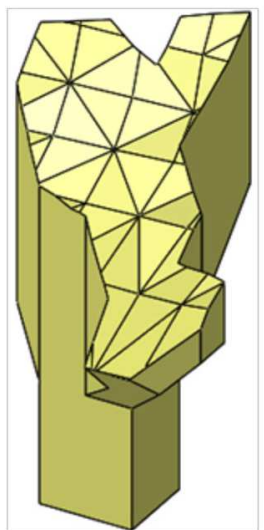

Right support, view from the surface to support

Fig. 14. Final part with its associated supports.

We demonstrated through a real case study that the methodology we propose is functional: it is possible, directly from the specifications, to get a CAD mock-up, including support, validating all requirements, ensuring the manufacturability and ready to undergo a slicing operation.

\section{Discussion}

Additive Manufacturing is present in both the industrial and domestic environments, and the process with the largest penetration level is the FDM. The expectations of these two categories of customers, however, remain similar: the production of quality parts. Three levers of action can be considered to obtain a part that meets the needs expressed in the specifications: the materials, the machine and the design of the part to be manufactured. We chose to focus on the design in order to propose a methodology to support designers in taking into account the specificities of the AM, from the constraints expressed by the specifications to the proposal of a digital mock-up ready to be manufactured on an AM machine.

We propose a methodology that takes into account AM specificities allowing the design of parts with complex geometries, composed of multiple materials, and which can incorporate fixed assemblies. This methodology has a structure that addresses comprehensively the lack of current DFAM methodologies: the material is no more imposed by the specifications, the validation of the part is possible before the phase of topological optimization, it is possible to change the result of topological optimization and the support is taken into account. Moreover, the integration of existing mathematical tools within the same CAD software allows to work with minimal disruptions in the digital chain. It remains two breaks, at the result of topological optimization and the result of discrete model of the part used as the basis for the generation of support. Currently, given the choices we have made, current tools do not allow to eliminate these two breaks.

To provide a turnkey solution, some steps of our methodology have to be automated. Currently, all the work needed to build the graph of functions and to determine the primary solid is done manually by the designer. Although this work cannot be replaced entirely by computer algorithms, it is still interesting to automate certain steps to reduce design time. For example, the creation of functional surfaces from the constraints of the specifications, the generation of the assembly of these surfaces and the verification of volumes of work could be done by the CAD software. Similarly, the principle of densification of surfaces, which aims to maximize the number of functional surfaces by manufacturable volume, consists simply in manually increasing the number of functional surfaces per set. It would therefore be possible to automate this step in order to request the CAD software a panel of proposed solutions after a multi objective optimization step which takes into account the working volumes of the different machines and the need for the separation of some surfaces. In this manner, the designer would select the most satisfactory result.

Similarly, for the second section of the methodology, the pre-orientation step, depending on the surface condition and the efforts required by the specifications, should ideally be performed by the software CAD using a multi objective optimization algorithm. The complexity of this step falls within the almost infinite possibilities of the part orientation if the dimensions of the part are very small compared to the working volume of the machine. Although the determination of the ideal orientation of the part is necessary for the preparation of the topological optimization, this step may require a long calculation time of about several hours. 
We focused here on the choice of the orientation of the part in order to optimize the surface quality or the solidity of the part. Studies demonstrated that the parts are stronger if the fibers are oriented in the load direction for tensile tests [11], or orthogonally to the yield load [30]. However, there are many parameters related to the manufacturing process or specific to the manufacturing choices (filling) that can have a significant influence on the part's resistance to compressive or tensile stresses (see [31] for an exhaustive review). Many studies have assessed the influence of the following factors: air gap, layer thickness, raster angle, raster width, deposition speed, temperature, and so on. Ahn et al. [11] have thus demonstrated that air gap and raster orientation affect strength. Onwubolu and Rayegani [9] carried out a study that highlighted an optimal combination of parameters to improve the tensile properties of the part: minimum layer thickness, negative air gap minimum raster width and increased raster angle. Other studies have tried to classify these different parameters according to their importance on the strength of the part [7]. Thus, to achieve a high level of strength, the consideration of manufacturing parameters in the FDM is one of the most critical design tasks [22]. The FDM process also brings additional constraints during manufacturing, due to the progressive cooling of the lower layers of the part being manufactured, causing a warping phenomenon, mainly in the corners. Here again, manufacturing and mesoscale parameters can have an impact on minimizing this phenomenon, for example by splitting pieces in hexagonal or squared bricks spatially locked [32], or by modulating layer thickness and extrusion velocity [33]. However, this phenomenon can be minimized simply by adopting a number of good practices: increase adhesion to the build plate, use a heated build plate, level correctly the build plate, add a brim or a raft, control cooling... In a future iteration, it would be interesting if our methodology could propose the use of a brim or a raft, depending on the dimensions and orientation of the part.

\section{Conclusion}

In conclusion, our proposal brings an original approach in the integration of business data and specifications in an easy to use tool, enabling a significant time saving for the designer. There are still many things to be taken into account, such as the control of manufacturing parameters that can lead to modulating the mechanical properties of the part and thus play a crucial role in the development of our methodology. Some constraints specific to the FDM process are only partially addressed here, such as taking into account the phenomenon of warping. We tried to propose a complete methodology, even if it can still be improved and will have to take into account more criteria and parameters to produce a part with the required strength while guaranteeing dimensional respect. Currently, in the finite element analysis, we consider the lowest value for Young's modulus for simplification purposes. In the future, less restrictive assumptions will have to be considered. Finally our methodology is restricted to the FDM process.
This work remains nevertheless a key step in achieving an integrated design methodology based on existing research, both in the areas of design, mathematics, mechanics, physics and chemistry. It also offers economic and industrial perspectives based on known and easy to implement tools such as CAD software, topological optimization and slicing software. In the short term, we wish to further automate and improve the performance of the ACSG method for support generation, as there is a lack (and an immediate need) in industry. The consideration of support accessibility is also an issue we want to improve. On a longer-term vision, we want to improve the transition between the specifications and the graph of functions, using a semantic analysis of technical terms. This would further increase the savings in time design and material consumption.

\section{References}

[1] P. Mognol, P. Muller, J.-Y. Hascoet, A novel approach to produce functionally graded materials for additive manufacturing, in Proceedings of the Conference on Advanced Research in Virtual and Rapid Prototyping, September 2011, pp. 473-478

[2] P. Muller, P. Mognol, J.-Y. Hascoët, Modeling and control of a direct laser powder deposition process for functionally graded materials (fgm) parts manufacturing, J. Mater. Process Technol. 213, 685-692 (2013)

[3] N. Boyard, M. Rivette, O. Christmann, S. Richir, Méthodologie de prise en compte de la fabrication additive lors de la phase de conception, in Colloque national AIP Primeca (13; 2012), Mont-dore, France, March 2012, pp. 1-13

[4] P. Kulkarni, A. Marsan, D. Dutta, A review of process planning techniques in layered manufacturing, Rapid Prototyp. J. 6, 18-35 (2000)

[5] O. Ivanova, C. Williams, T. Campbell, Additive manufacturing (am) and nanotechnology: promises and challenges, Rapid Prototyp. J. 19, 353-364 (2013)

[6] M. Dawoud, I. Taha, S.J. Ebeid, Mechanical behaviour of abs: An experimental study using fdm and injection moulding techniques, J. Manuf. Process 21, 39-45 (2016)

[7] A.W. Gebisa, H.G. Lemu, Investigating effects of fuseddeposition modeling $(\mathrm{fdm})$ processing parameters on flexural properties of ultem 9085 using designed experiment, Materials (Basel) 11 (2018) 500

[8] A.K. Sood, R.K. Ohdar, S.S. Mahapatra, Experimental investigation and empirical modelling of fdm process for compressive strength improvement, J. Adv. Res. 3, 81-90 (2012)

[9] G.C. Onwubolu, F. Rayegani, Characterization and optimization of mechanical properties of abs parts manufactured by the fused deposition modelling process, Int. J. Manuf. Eng. 2014, 13 (2014)

[10] J.M. Chacón, M.Á. Caminero, E. García-Plaza, P.J. Núñez, Additive manufacturing of pla structures using fused deposition modelling: Effect of process parameters on mechanical properties and their optimal selection, Mater. Des. 124, 143-157 (2017)

[11] S.-H. Ahn, M. Montero, D. Odell, S. Roundy, P.K. Wright, Anisotropic material properties of fused deposition modeling abs, Rapid Prototyp. J. 8, 248-257 (2002) 
[12] K.P. Motaparti, G.Taylor, M.C. Leu, K. Chandrashekhara, J. Castle, M. Matlack, Effects of build parameters on compression properties for ultem 9085 parts by fused deposition modeling, in Proceedings of the 27th Annual International Solid Freeform Fabrication Symposium, Austin, Texas, USA, 2016, pp. 8-10

[13] M. Fernandez-Vicente, W. Calle, S. Ferrandiz, A. Conejero, Effect of infill parameters on tensile mechanical behavior in desktop 3D printing, 3D Print Addit. Manuf. 3, 183-192 (2016)

[14] T.J. Howard, S.J. Culley, E. Dekoninck, Describing the creative design process by the integration of engineering design and cognitive psychology literature, Des. Stud. 29, 160-180 (2008)

[15] G. Pahl, W. Beitz, J.A. Feldhusen, K.-H Grote, Engineering Design: A Systematic Approach, Springer-Verlag, London, UK, 2007

[16] C. Hales, S. Gooch, Managing Engineering Design, Springer, London, UK, 2004

[17] V.D. Ingenieure, Konstruktionsmethodik Methodisches Entwickeln von Lösungsprinzipien Blatt 1, Beuth Düsseldorf, Berlin, Deutschland, 1997

[18] H. Rodrigue, Méthodologie de conception et d'optimisation de mécanismes fabriqués par fabrication rapide, $\mathrm{PhD}$ thesis, Ecole Polytechnique de Montréal, Canada, 2010

[19] J.-Y. Hascoët, R. Ponche, O. Kerbrat, P. Mognol, From functional specifications to optimized cad model: Proposition of a new dfam methodology, in T.M.D. Ferreira (ed.), International Conference on Advanced Research in Virtual and Rapid Prototyping, 2011

[20] O. Kerbrat, P. Mognol, J.-Y. Hascoët, A new dfm approach to combine machining and additive manufacturing, Comput. Ind. 62, 684-692 (2011)

[21] R. Ponche, Méthodologie de conception pour la fabrication additive, application $\tilde{\mathrm{A}}$ la projection de poudre, $\mathrm{PhD}$ thesis, Ecole Centrale de Nantes, France, 2013

[22] U.K. uz Zaman, E. Boesch, A. Siadat, M. Rivette, A.A. Baqai, Impact of fused deposition modeling (fdm) process parameters on strength of built parts using taguchi's design of experiments, Int. J. Adv. Manuf. Technol. 101, 12151226 (2019)

[23] K. Singh, Experimental study to prevent the warping of 3d models in fused deposition modeling, Int. J. Plast. Technol. 22, 177-184 (2018)

[24] W. Yu, Electre tri(aspects méthodologiques et manuel d'utilisation), Document- Université de Paris-Dauphine, LAMSADE, 1992

[25] J.-H. Kao, F.B. Prinz, Optimal motion planning for deposition in layered manufacturing, in Proceedings of Design Engineering Technical Conference, 1998, pp. 13-16

[26] C. Fonda, A practical guide to your first 3D print, Low-cost 3D printing for science, education and sustainable development, 1st edn. ICTP/he Abdus Salam International Centre for Theoretical Physica, Trieste, Italy, 2013, pp. 25-60

[27] C.B. Williams, J.K. Cochran, D.W. Rosen, Additive manufacturing of metallic cellular materials via threedimensional printing, Int. J. Adv. Manuf. Technol. 53, 231-239 (2011)

[28] G. Strano, L. Hao, R.M. Everson, K.E. Evans, A new approach to the design and optimisation of support structures in additive manufacturing, Int. J. Adv. Manuf. Technol. 66, 1247-1254 (2013)

[29] N. Boyard, Méthodologie de conception pour la réalisation de pièces en fabrication additive, $\mathrm{PhD}$ thesis, Ecole Nationale Supérieure d'Arts et Métiers, Paris, France, 2013

[30] A.K. Sood, R.K. Ohdar, S.S. Mahapatra, Parametric appraisal of mechanical property of fused deposition modelling processed parts, Mater. Des. 31, 287-295 (2010)

[31] O.A. Mohamed, S.H. Masood, J.L. Bhowmik, Optimization of fused deposition modeling process parameters: a review of current research and future prospects, Adv. Manuf. 3, 42-53 (2015)

[32] A. Guerrero de Mier, M.M. Espinosa, M. Domínguez, Bricking: A new slicing method to reduce warping, Proc. Manuf. 132, 126-131 (2015)

[33] B.N. Panda, K. Shankhwar, A. Garg, Z. Jian, Performance evaluation of warping characteristic of fused deposition modelling process, Int. J. Adv. Manuf. Technol. 88, 1799-1811 (2017)

Cite this article as: Boyard Nicolas, Christmann Olivier, Rivette Mickael and Richir Simon, A design methodology for additive manufacturing applied to fused deposition modeling process, Mechanics \& Industry 20, 608 (2019) 